“C 2017 IEEE. Personal use of this material is permitted. Permission from IEEE must be obtained for all other uses, in any current or future media, including reprinting/republishing this material for advertising or promotional purposes, creating new collective works, for resale or redistribution to servers or lists, or reuse of any copyrighted component of this work in other works." 


\title{
Hybrid Power Plant Offering Strategy to Deal with the Stochastic Nature and Outage of Wind Generators
}

\author{
Sahand Ghavidel ${ }^{1 *}$, Mostafa Barani ${ }^{2}$, Ali Azizivahed ${ }^{2}$, Li Li $^{1}$, and Jiangfeng Zhang ${ }^{1}$ \\ ${ }^{1}$ Faculty of Engineering and Information Technology, University of Technology Sydney, PO Box 123, Broadway, NSW 2007, \\ Australia \\ ${ }^{2}$ Department of Electrical and Electronics Engineering, Shiraz University of Technology, Shiraz, Iran \\ 1*Email: sahand.ghavideljirsaraie@ student.uts.edu.au
}

\begin{abstract}
The stochastic nature of wind power generators and their possible outage are crucial issues which make them difficult to participate in electricity markets. However, demand side as a decent balancing resource can be used to compensate the challenges of lack of supply-demand balance or state of outage for wind generators. This paper firstly models the outage of wind generators. Then an offering strategy with a three-stage stochastic programming is presented for a hybrid power plant which includes a wind power producer and a demand response provider. Three electricity markets are considered including day-ahead, adjustment and balancing market. The conditional value-atrisk is also added to the offering strategy to control the profit risk. The offering strategy is tested in a wind farm and electricity market located in Spain. The result shows that the hybrid power plant offering strategy can effectively assist with the balancing and outage problem of the wind power producer and increase the overall profit of the joint operation.
\end{abstract}

Index Terms-- Hybrid power plant, Wind power producer, Demand response provider, Conditional value-at-risk, Stochastic programming.

\section{INTRODUCTION}

\section{A. Aims and Scope}

Due to the stochastic, unstable and undispatchable nature of wind generations and their possible outage, it is usually very difficult for these kinds of producers to take part in electricity markets and rival other producers such as conventional power plants. Accordingly, it is vital to propose a new strategy for wind power producers to assist them to tackle these problems [1].

Recently, using demand response programs as a rapid resource generator has been rapidly growing for different purposes [2, 3]. The ability to rapidly respond makes demand response resources as a flexible resource to manage the unstable nature or even possible outage of wind power generators $[4,5]$.

\section{B. Literature Review and Approach}

Many studies provide an offering strategy for wind power producers to participate in electricity markets [6-8]. For example, Ref. [9] offers a procedure to develop an offering strategy for a wind power producer including different types of electricity markets and considering the uncertainty related to stochastic nature of wind and prices of different markets. Ref. [10] recommends an offering strategy by considering a wind power producer as a pricemaker in the day-ahead (DA) market. In this paper, the uncertainties related to stochastic nature of wind and prices of different markets are also considered. Ref. [11] studies an offering strategy of a wind power producer as a pricetaker in the DA market and a price-maker in the balancing market. Ref. [12] considers two different offering strategies for cooperative contribution of a wind power generator in two electricity markets including energy and primary reserve markets.

Also, some studies provide offering strategies for wind power producers along with other producers [13-15]. For example, Ref. [16] studies the joint operation of a wind power producer and a pumped-storage facility by considering the uncertainty related to stochastic nature of wind and market prices. The impact of stochastic nature of wind on the amount of pumped-hydro stored energy in a future U.K. system is evaluated in [17]. Ref. [18] offers a bidding strategies for a wind power producer and hydro facility to be able to participate in a DA market using conditional value-at-risk model to control the financial risk. Ref. [19] evaluates two models including a wind power producer supported with a gas turbine, and a wind power producer supported with a compressed air energy storage.

Recently, many studies offer demand response resources as a more flexible resource to manage the unstable nature of wind power generators [20-22]. For example, Ref. [23] specifies the optimized value of load as a demand response for congestion management and improving the use of wind power generations. An offering strategy for a wind power producer and a flexible load which is able to cover the wind power imbalances is suggested in [24] for participation in a DA electricity market. In order to minimize the total operational cost including imbalance fines because of wind energy over- and under-commitments, an optimal scheduling of critical peak pricing events is evaluated in [25] from the perspective of an demand response facility which has wind energy to properly trade into the DA market. Ref. [26] offers a new offering strategy for a wind power producer to participate in three electricity markets including DA, intraday and balancing markets with the help of a demand response resource which is allowed to contribute to the intraday market. Ref. [27] offers a novel method considering the uncertainty related to the amount of wind power generation and the load for corrective voltage control to cope with situation that a power system 


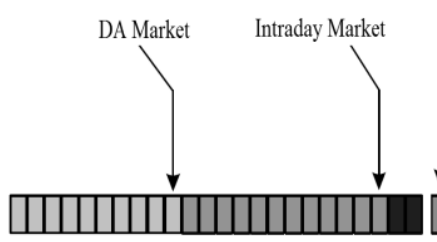

Current operating day: Day D

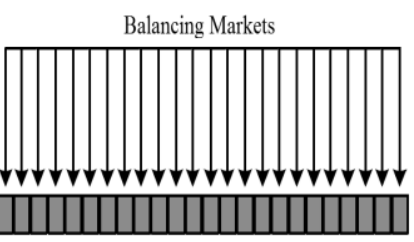

Next operating day: Day D+1
Fig. 1 Electricity markets structure.

meets voltage instability due to severe contingencies.

This paper proposes an approach for the joint operation of a wind power producer and a demand response provider as a hybrid power plant in which the wind power producer utilizes the demand response provider as a storage facility. The hybrid power plant participates in the DA, intraday (adjustment), and balancing markets. The uncertainties of wind generator production, their outage, and market prices of three markets are considered. In order to find the best offering strategy, the stochastic programming method is used, and then the conditional value-at-risk is added to control the financial risk.

\section{Paper Organization}

The rest of the paper is organized as follows. Section II formulates the offering strategy of the hybrid power plant. Section III provides the results and discuses about the outcomes for the hybrid strategy. Finally, Section IV reviews the results of this study and draws conclusions.

\section{PROBLEM Formulation}

In this section, the offering strategy of the hybrid power plant is modeled. Three electricity markets are considered in this paper as shown in Fig. 1. The model of the hybrid power plant including these three electricity markets can be formulated as a maximization problem which is composed of maximization of the revenue of the hybrid power plant minus its total cost. Therefore, the objective function can be written as follows [20]:

$$
\begin{gathered}
\sum_{\omega=1}^{N_{\Omega}} \sum_{t=1}^{N_{T}} \rho_{\omega}\left[v_{t \omega}^{D} \cdot P_{t \omega}^{D, H p p}+v_{t \omega}^{I} \cdot P_{t \omega}^{I, H p p}+\right. \\
\left.\frac{1}{2} \cdot \frac{1}{\sigma \cdot D_{0 t}} \cdot\left(L_{t \omega}^{s c h}\right)^{2}+v_{t \omega}^{D} \cdot R_{t \omega}^{+} \cdot \delta_{t \omega}^{+, H p p}-v_{t \omega}^{D} \cdot R_{t \omega}^{-} \cdot \delta_{t \omega}^{-, H p p}\right]+ \\
\beta\left(\lambda-\frac{1}{(1-\alpha)} \sum_{\omega=1}^{N_{\Omega}} \rho_{\omega} Z_{\omega}\right) .
\end{gathered}
$$

In equation (1), $t$ and $N_{T}$ are the index for hourly periods and the total period of time, respectively. $N_{\Omega}$ is the total number of scenarios. $v_{t \omega}^{D}$ and $v_{t \omega}^{I}$ are the DA and intra-day market prices, respectively. $P_{t \omega}^{D, H p p}$ and $P_{t \omega}^{I, H p p}$ are DA and intra-day offers of the hybrid power plant (superscript $H p p$ stands for hybrid power plant). $\sigma$ is a factor that defines the relationship between elasticity and price. $D_{0 t}$ is the normal level of demand in period $t . L_{t \omega}^{s c h}$ is the scheduled power of the demand side. $R_{t \omega}^{+}$and $R_{t \omega}^{-}$are the positive and negative imbalance price ratios. $\delta_{t \omega}^{+, H p p}$ and $\delta_{t \omega}^{-, H p p}$ are the positive and negative deviations of the hybrid power plant from the scheduled power. The last term of the equation (1) is related to modeling of the conditional value-at-risk. In this term, $\beta$ is the risk-aversion factor. By increasing the value of $\beta$, the financial risk will be decreased. $\lambda$ and $z_{\omega}$ are supplementary variables to calculate the conditional value- at-risk. $\alpha$ is the confidence level. Also, $\rho_{\omega}$ is the probability of occurrence of scenario $\omega$.

The objective function defined in equation (1) is subject to some constraints which include some combined constraints related to both wind power producer and demand response provider and some constraints related to modelling the flexible load only.

The offer limitation of the hybrid power plant to the DA market can be written as follows:

$$
0 \leq P_{t \omega}^{D, H p p} \leq W^{\max }+\eta_{1} D_{0 t} \quad \forall t, \forall \omega
$$

where $W^{\max }$ is the wind producer capacity and $\eta_{1} D_{0 t}$ is the demand response provider capacity. The reason that minimum of the constraint (2) is equal to zero is that the hybrid power plant is considered to be a generation company.

The constraint on the total scheduled power of DA and intra-day offers can be formulated as follows:

$$
P_{t \omega}^{s c h, H p p}=P_{t \omega}^{D, H p p}+P_{t \omega}^{I, H p p} \quad \forall t, \forall \omega
$$

where $P_{t \omega}^{s c h, H p p}$ is the scheduled power of hybrid power plant aggregator.

The scheduled power limitation of the hybrid power plant can be written as follows:

$$
0 \leq P_{t \omega}^{s c h, H p p} \leq W^{\max }+\eta_{1} D_{0 t} \quad \forall t, \forall \omega
$$

The total, negative, and positive imbalances of the hybrid power plant based on the wind power production, scheduled power and the values of demand response can be characterized as the following constraints:

$$
\begin{aligned}
& \delta_{t \omega}^{H p p}=W_{t \omega}+L_{t \omega}-P_{t \omega}^{s c h, H p p} \quad \forall t, \forall \omega \\
& \delta_{t \omega}^{H p p}=\delta_{t \omega}^{+, H p p}-\delta_{t \omega}^{-, H p p} \quad \forall t, \forall \omega \\
& 0 \leq \delta_{t \omega}^{+, H p p} \leq W_{t \omega}+L_{t \omega} \quad \forall t, \forall \omega \\
& 0 \leq \delta_{t \omega}^{-, H p p} \leq W^{\max }+\eta_{1} D_{0 t} \quad \forall t, \forall \omega
\end{aligned}
$$

where $W_{t \omega}$ and $L_{t \omega}$ are the active wind power production and demand response provider power that will be finally delivered. In this paper, it is assumed that active power of demand response provider and its scheduled value are equal, that is, there is no uncertainty in the case of demand response provider production. The intra-day offer limitation with respect to the DA offer can be defined as follows:

$-\gamma \cdot P_{t \omega}^{D, H p p} \leq P_{t \omega}^{I, H p p} \leq \gamma \cdot P_{t \omega}^{D, H p p} \quad \forall t, \forall \omega$ where $\gamma$ is a factor for limiting offers to the intra-day market.

For calculation of risk, the following constraints are required.

$$
\begin{aligned}
& -\sum_{t=1}^{N_{T}}\left[v_{t \omega}^{D} \cdot P_{t \omega}^{D, H p p}+v_{t \omega}^{I} P_{t \omega}^{I, H p p}+\frac{1}{2} \cdot \frac{1}{\sigma \cdot D_{0 t}} \cdot\left(L_{t \omega}^{s c h}\right)^{2}+\right. \\
& \left.v_{t \omega}^{D} \cdot R_{t \omega}^{+} \cdot \delta_{t \omega}^{+, H p p}-v_{t \omega}^{D} \cdot R_{t \omega}^{-} \cdot \delta_{t \omega}^{-, H p p}\right]+\lambda-z_{\omega} \leq 0 \\
& z_{\omega} \geq 0 \quad \forall \omega
\end{aligned}
$$




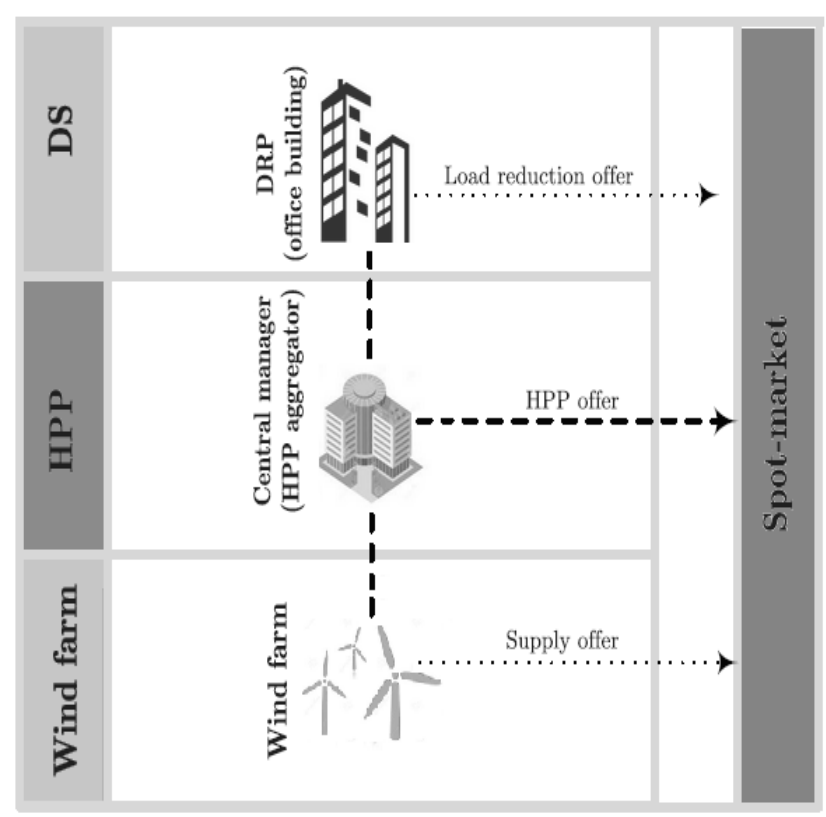

Fig. 2 Three different configurations: demand respond provider only, wind power producer only, and hybrid power plant.

The constraints related to modeling flexible load can be defined as following constraints:

$$
\begin{aligned}
& L_{t \omega}^{s c h}=L_{t}^{D}+L_{t \omega}^{I} \quad \forall t, \forall \omega \\
& \eta_{2} D_{0 t} \leq L_{t}^{D} \leq \eta_{1} D_{0 t} \quad \forall t, \forall \omega \\
& \eta_{2} D_{0 t} \leq L_{t \omega}^{s c h} \leq \eta_{1} D_{0 t} \quad \forall t, \forall \omega \\
& \sum_{t=1}^{N_{T}} L_{t \omega}^{s c h} \leq \mu \cdot \sum_{t=1}^{N_{T}} D_{0 t} \quad \forall \omega \\
& L_{t \omega}^{s c h}=L_{t \omega^{\prime}}^{s c h} \quad \forall t, \forall \omega, \forall \omega^{\prime}: v_{t \omega}^{D}=v_{t \omega^{\prime}}^{D} \\
& L_{t \omega}^{I}=L_{t \omega^{\prime}}^{I} \quad \forall t, \forall \omega, \forall \omega^{\prime}: v_{t \omega}^{D}=v_{t \omega^{\prime}}^{D}
\end{aligned}
$$

where $L_{t}^{D}$ is the amount of the load reduction to offer to DA market in period t. $L_{t \omega}^{I}$ is the amount of the load reduction to offer to the intra-day market in period $t$ and scenario $\omega$. $\mu$ is the maximum percentage of the load which is not required to be recovered. Constraints (16) and (17) express the non-anticipativity of the decision in the intra-day market. The lower limits of constraints (13)-(14) is decreased from zero to $\eta_{2} D_{0 t}\left(\eta_{2}<0\right)$ because of the fact that demand response can exploit wind energy and increases its load in the joint operation.

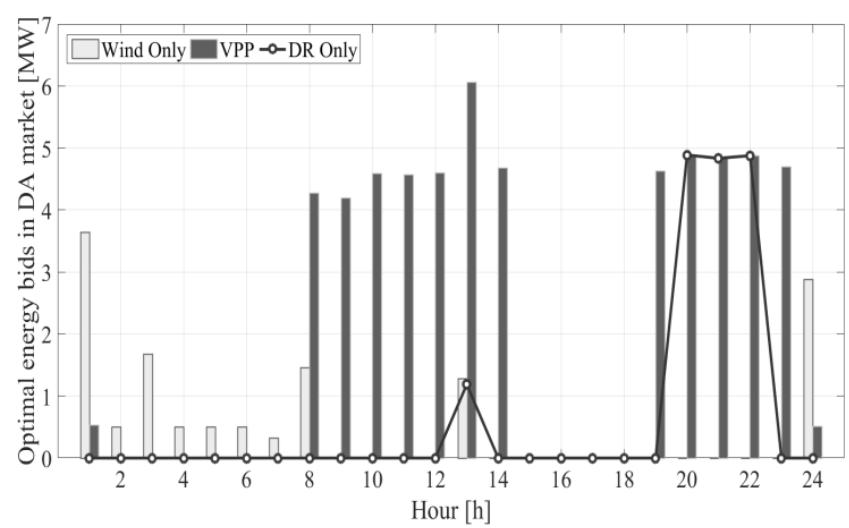

Fig. 3 Optimal offering bids in the DA market
TABLE I

PROBABILITY DISTRIBUTIONS OF THE OUTAGE TIME OF THE WIND GENERATION

\begin{tabular}{ccccc}
\hline \hline \multirow{2}{*}{ Parameters } & \multicolumn{4}{c}{ Probability Distribution Characteristics } \\
\cline { 2 - 5 } & Mean & St. Dev. & Min. & Max. \\
\hline$T^{\text {Start }}$ & 13.5 & 1 & 11 & 16 \\
\hline$T^{\text {Stop }}$ & 20 & 1 & 17 & 23
\end{tabular}

For proposing non-decreasing offering curves to DA market, the following constraints can be defined:

$$
\begin{aligned}
& \left(P_{t \omega}^{D, H p p}-P_{t \omega^{\prime}}^{D, H p p}\right) \cdot\left(v_{t \omega}^{D}-v_{t \omega^{\prime}}^{D}\right) \geq 0 \quad \forall t, \forall \omega, \forall \omega^{\prime} \\
& P_{t \omega}^{D, H p p}=P_{t \omega^{\prime}}^{D, H p p} \quad \forall t, \forall \omega, \forall \omega^{\prime}: v_{t \omega}^{D}=v_{t \omega^{\prime}}^{D}
\end{aligned}
$$

\section{RESULTS AND DISCUSSIONS}

The proposed methodology aims to coordinate a wind power producer and a demand response provider in order to tackle the uncertainties related to electricity markets prices, wind power and its outage. Three different configurations are simulated including demand respond provider only, wind power producer only, and hybrid power plant as shown in Fig. 2.

Wind power and market price uncertainties are modelled and generated with a set of scenarios using an adapted hybrid neural network and improved particle swarm optimization. The scenarios related to severe outages of the wind generators are generated according to the statistical analysis.

The suggested method is applied on a real case study in the Sotavento wind farm with 17.56 MW capacity in Spain [28]. The stochastic input of the wind power generation is modelled with a process presented in [20]. In order to train the artificial neural network, the historical data of 2010 are used. The scenarios related to market prices are derived by a three-step process as presented in [29]. The historical data of demand and market prices are also derived based on the Iberian Peninsula electricity market [30]. The uncertainties of the problem are modelled through a scenario tree with 3000 scenarios $(10 \times 5 \times 10 \times 6)$ including ten, five, ten and, six scenarios for DA, intra-day, balancing market prices and the wind generation, respectively. The outage time of the wind generation is randomly generated. The probability distributions of the outage time of the wind generation are specified in Table I. In this table, $T^{\text {Start }}$ stands for the beginning time of the wind generation outage time and $T^{\text {Stop }}$ stands for the ending time of the wind generation outage time. These outage times are added to the previously produced wind power stochastic profile as a zero power replacement.

The simulation results are presented for 12th of March, 2010. It is presumed that 0.067 percent of the total loads of the Spanish electricity market are combined to participate in the market through a demand response provider.

The proposed model is firstly implemented in MATLAB for the scenario generation and reduction, and then these data are brought in GAMS by means of GAMS/MATLAB interface to solve the optimization 


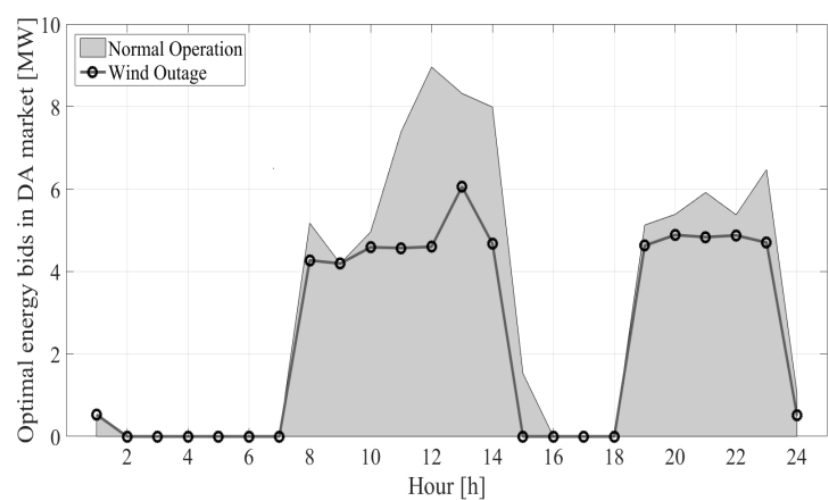

Fig. 4 Hybrid power plant optimal energy bids in DA market in two different conditions: normal operation and wind outage.

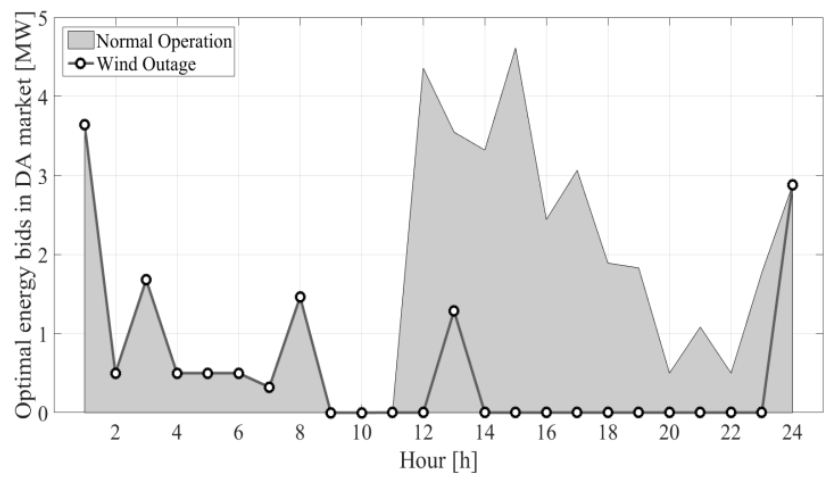

Fig. 5 Independent wind power producer optimal energy bids in DA market in two different conditions: normal operation and wind outage.

problem. Note that the problem is solved using CPLEX under GAMS, and the simulations are done in 120.756 seconds on a $2.3 \mathrm{GHz}$ Intel® $\mathrm{CORE}^{\mathrm{TM}}$ i5 laptop with $8 \mathrm{~GB}$ of RAM.

Fig. 3 shows the comparison of energy hourly bids into the DA market in wind outage condition for scenario number 5 among three different configurations: wind power producer only, demand response provider only, and hybrid power plant. As can be seen, the demand response provider stores more energy during off-peak hours in the joint operation with the help of the wind power producer, but the demand response provider releases the stored energy to the market in the peak hours in the independent operation. Moreover, the joint operation strategy achieves $€ 96.171$ extra profits in comparison with the independent operation of wind power producer and demand response provider.

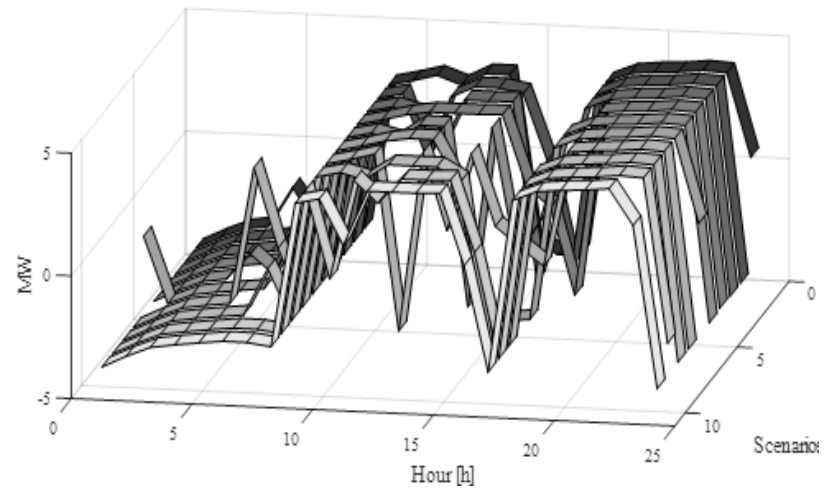

Fig. 6 Optimal variations in the behaviour of the demand response provider for each scenario in the joint operation.

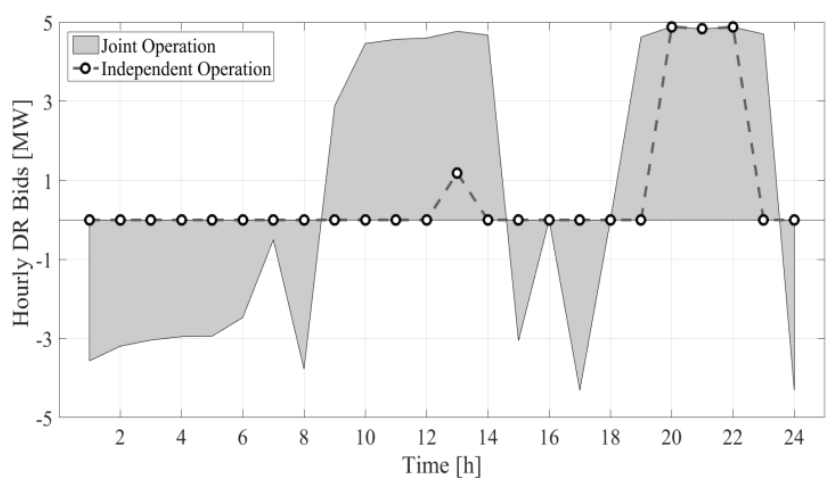

Fig. 7 Optimal offering bids of demand response provider in DA market in two conditions: Joint operation and independent operation.

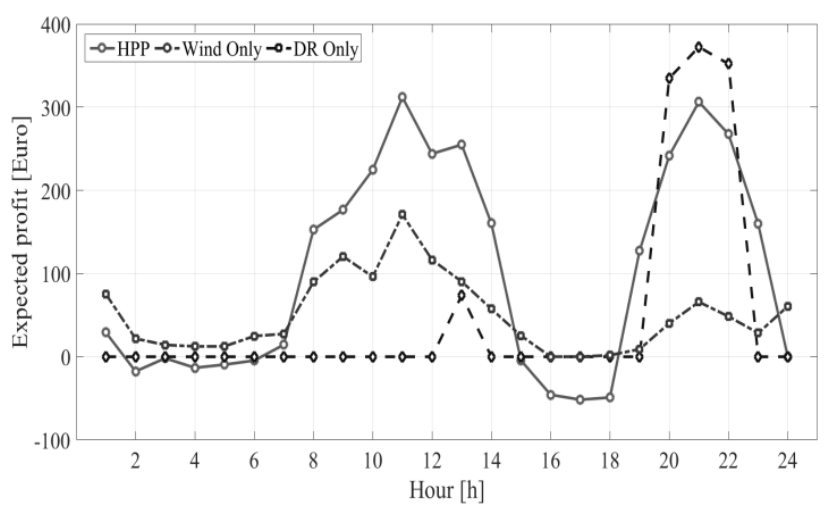

Fig. 8 Comparison of hourly expected profit under the three configurations: demand respond provider only, wind power producer only, and hybrid power plant.

Also, Fig. 4 and Fig. 5 show optimal energy bids for scenario number 5 in DA market of the hybrid power plant and independent wind power producer in two different conditions: normal operation and wind outage. As it can be seen from these figures, in the wind outage condition, the independent wind power has no offering in most hours including hours 9-12 and hours 14-23. On the other hand, the hybrid power plant has slightly less offering energy to DA market in the wind outage condition in comparison of the normal condition.

Fig. 6 shows the optimal variations in the behaviour of the demand response provider for each scenario in the joint operation for DA market. As it can be seen from this figure, the demand response provider has different responses corresponding to different DA market scenarios. Also, optimal offering bids of demand response

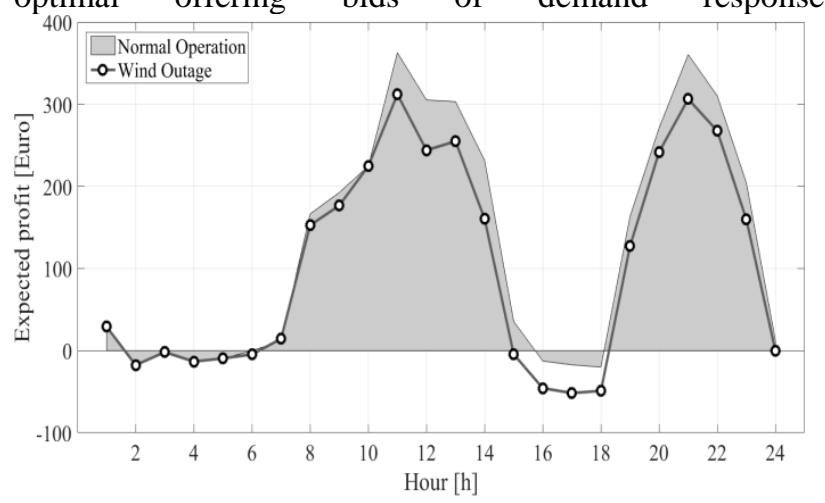

Fig. 9 Hybrid power plant hourly expected profit in two different conditions: normal operation and wind outage. 


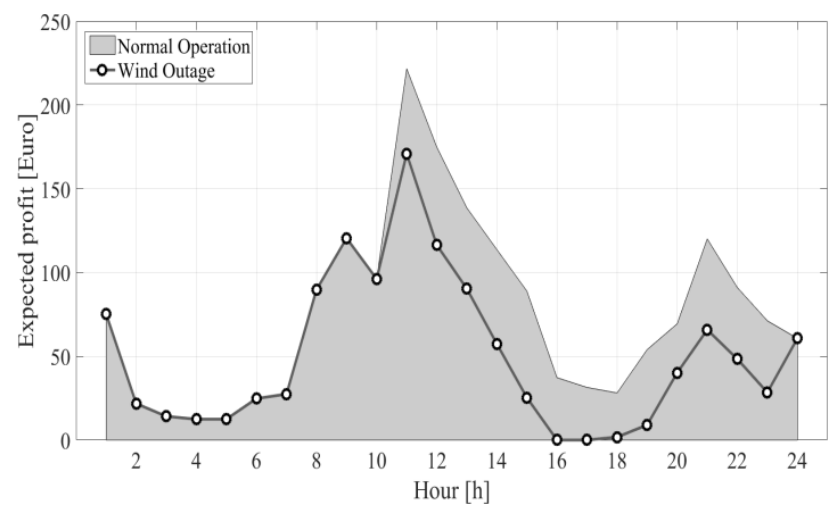

Fig. 10 Independent wind power producer hourly expected profit in two different conditions: normal operation and wind outage.

provider in DA market in two conditions: joint operation and independent operation is shown in Fig. 7. As it can be seen from this figure, by coordinating with wind power producer, the demand response provider can be fully used and engaged in the system.

Fig. 8 shows the comparison of hourly expected profit under the three configurations in the wind outage condition.

Also, Fig. 9 and Fig. 10 show hourly expected profit of the hybrid power plant and independent wind power producer in two different conditions: normal operation and wind outage.

Fig. 11 and Fig. 12 show the changing pattern of independent wind power producer and hybrid power plant
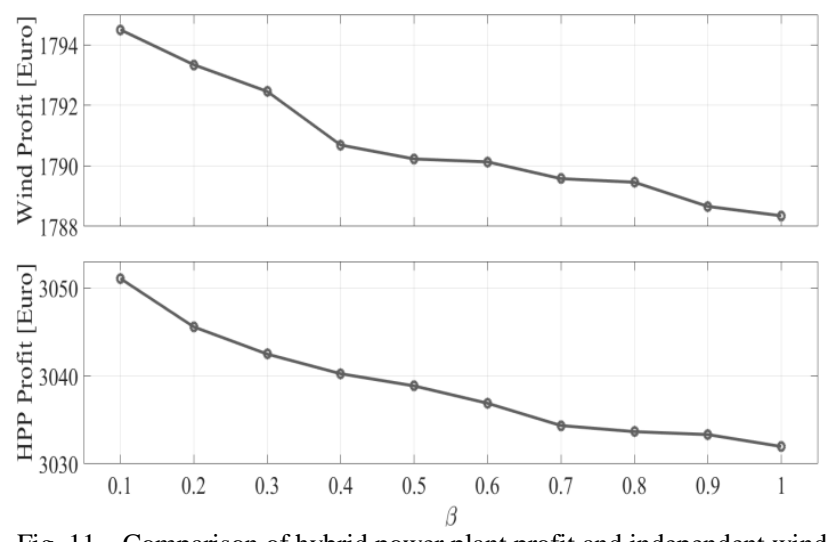

Fig. 11 Comparison of hybrid power plant profit and independent wind power producer profit for different values of $\beta$ in the normal operation condition.
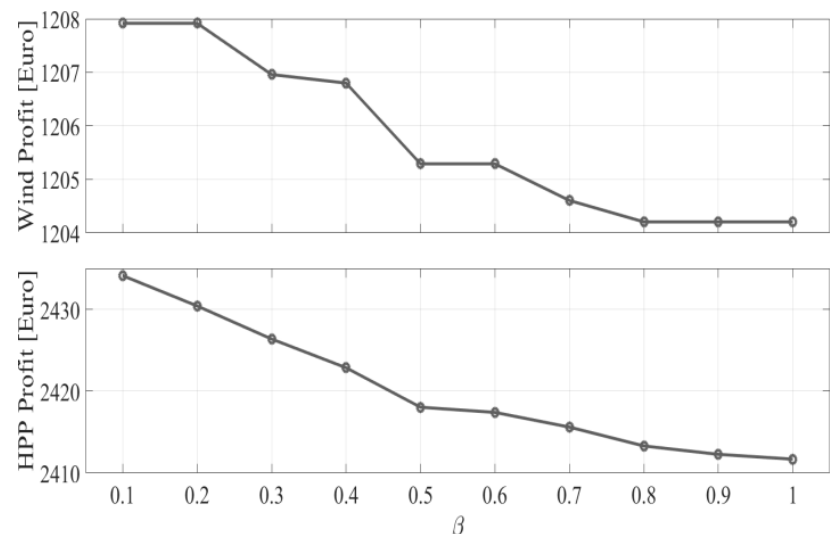

Fig. 12 Comparison of hybrid power plant profit and independent wind power producer profit for different values of $\beta$ in the wind outage condition.
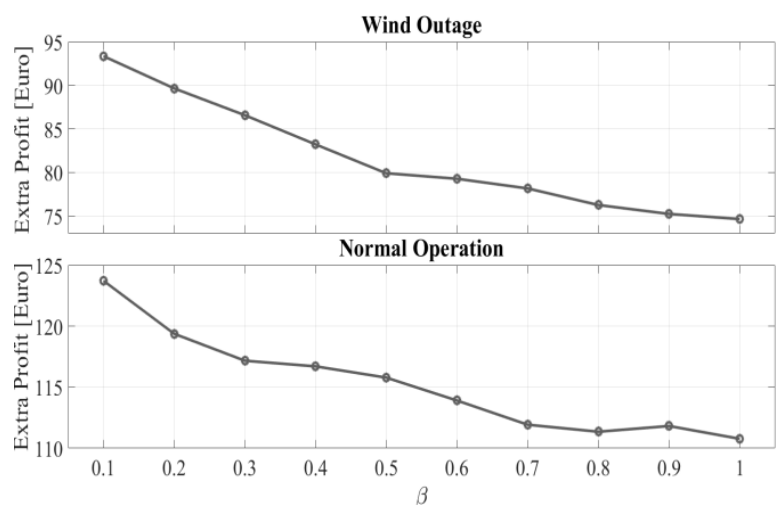

Fig. 13 Comparison of hybrid power plant extra profit for different values of $\beta$ in two conditions: normal operation and wind outage.

profits versus different values of the risk factor $\beta$. As it can be seen in these figures, by increasing the risk factor $\beta$, the profits related to independent wind power producer and hybrid power plant in both conditions: normal operation and wind outage are decreased. However, these decreases of the profits are reasonable because the financial risk in the system will be decreased as well. The extra profit of using hybrid power plant over independent operation for different values of of the risk factor $\beta$ is shown in Fig. 13. As it can be seen in this figure, the extra profit tends to decrease with the increasing $\beta$. Note that, the maximum extra profit (i.e. equal to 124.056) is achieved at risk factor $\beta=0.1$ in the normal operation condition, and the minimum extra profit (i.e. equal to 74.986) is achieved at $\beta$ $=0.9$ in the wind outage condition.

\section{CONCLUSION}

In this study, a wind power producer and a demand response provider have been coordinated as a hybrid power plant to participate in three electricity markets including DA, intra-day and balancing markets. The uncertainties related to electricity market prices, the wind power and its outage have been considered in the paper.

The uncertainty related to the wind outage has been randomly produced and added to the wind uncertainties in the normal operation. The problem has been formulated as a three-stage stochastic programming which includes DA market as the first stage, intraday market as the second stage and finally balancing market as the third stage. In order to control the financial risk, the conditional value-atrisk has been added to the formulation.

\section{REFERENCES}

T. Dai and W. Qiao, "Finding equilibria in the poolbased electricity market with strategic wind power producers and network constraints," IEEE Transactions on Power Systems, vol. 32, pp. 389-399, 2017.

R. H. Auba, G. Wenzel, D. Olivares, and M. NegretePincetic, "Participation of Demand Response Aggregators in Electricity Markets: Optimal Portfolio Management," IEEE Transactions on Smart Grid, 2017. N. Mahmoudi, E. Heydarian-Forushani, M. Shafiekhah, T. K. Saha, M. Golshan, and P. Siano, "A bottomup approach for demand response aggregators' 
participation in electricity markets," Electric Power Systems Research, vol. 143, pp. 121-129, 2017.

[4] A. Dolatabadi and B. Mohammadi-Ivatloo, "Stochastic risk-constrained scheduling of smart energy hub in the presence of wind power and demand response," Applied Thermal Engineering, vol. 123, pp. 40-49, 2017.

[5] Y. Jiang, J. Xu, Y. Sun, C. Wei, J. Wang, D. Ke, et al., "Day-ahead stochastic economic dispatch of wind integrated power system considering demand response of residential hybrid energy system," Applied Energy, vol. 190, pp. 1126-1137, 2017.

[6] L. Baringo and A. J. Conejo, "Offering strategy of windpower producer: A multi-stage risk-constrained approach," IEEE Transactions on Power Systems, vol. 31, pp. 1420-1429, 2016.

[7] H. M. I. Pousinho, V. M. F. Mendes, and J. P. d. S. Catalão, "A stochastic programming approach for the development of offering strategies for a wind power producer," Electric Power Systems Research, vol. 89, pp. 45-53, 2012.

[8] M. Hosseini-Firouz, "Optimal offering strategy considering the risk management for wind power producers in electricity market," International Journal of Electrical Power \& Energy Systems, vol. 49, pp. 359368, 2013.

[9] J. M. Morales, A. J. Conejo, and J. Pérez-Ruiz, "Shortterm trading for a wind power producer," IEEE Transactions on Power Systems, vol. 25, pp. 554-564, 2010.

[10] L. Baringo and A. J. Conejo, "Strategic offering for a wind power producer," IEEE Transactions on Power Systems, vol. 28, pp. 4645-4654, 2013.

[11] M. Zugno, J. M. Morales, P. Pinson, and H. Madsen, "Pool strategy of a price-maker wind power producer," IEEE Transactions on Power Systems, vol. 28, pp. 34403450, 2013.

[12] T. Soares, P. Pinson, T. V. Jensen, and H. Morais, "Optimal offering strategies for wind power in energy and primary reserve markets," IEEE Transactions on Sustainable Energy, vol. 7, pp. 1036-1045, 2016.

[13] H. Ding, P. Pinson, Z. Hu, and Y. Song, "Optimal offering and operating strategies for wind-storage systems with linear decision rules," IEEE Transactions on Power Systems, vol. 31, pp. 4755-4764, 2016.

[14] H. Ding, P. Pinson, Z. Hu, J. Wang, and Y. Song, "Optimal Offering and Operating Strategy for a Large Wind-Storage System as a Price Maker," IEEE Transactions on Power Systems, 2017.

[15] H. Ding, P. Pinson, Z. Hu, and Y. Song, "Integrated bidding and operating strategies for wind-storage systems," IEEE Transactions on Sustainable Energy, vol. 7, pp. 163-172, 2016.

[16] J. Garcia-Gonzalez, R. M. R. de la Muela, L. M. Santos, and A. M. Gonzalez, "Stochastic joint optimization of wind generation and pumped-storage units in an electricity market," IEEE Transactions on Power Systems, vol. 23, pp. 460-468, 2008.

[17] M. Black and G. Strbac, "Value of bulk energy storage for managing wind power fluctuations," IEEE transactions on energy conversion, vol. 22, pp. 197-205, 2007.

[18] A. A. S. de la Nieta, J. Contreras, and J. I. Munoz, "Optimal coordinated wind-hydro bidding strategies in day-ahead markets," IEEE Transactions on Power Systems, vol. 28, pp. 798-809, 2013.
[19] J. B. Greenblatt, S. Succar, D. C. Denkenberger, R. H. Williams, and R. H. Socolow, "Baseload wind energy: modeling the competition between gas turbines and compressed air energy storage for supplemental generation," Energy Policy, vol. 35, pp. 1474-1492, 2007.

[20] J. Aghaei, M. Barani, M. Shafie-Khah, A. A. S. de la Nieta, and J. P. Catalão, "Risk-constrained offering strategy for aggregated hybrid power plant including wind power producer and demand response provider," IEEE Transactions on Sustainable Energy, vol. 7, pp. 513-525, 2016.

[21] M. Asensio and J. Contreras, "Risk-constrained optimal bidding strategy for pairing of wind and demand response resources," IEEE Transactions on Smart Grid, vol. 8, pp. 200-208, 2017.

[22] X. Fang, Q. Hu, F. Li, B. Wang, and Y. Li, "Couponbased demand response considering wind power uncertainty: a strategic bidding model for load serving entities," IEEE Transactions on Power Systems, vol. 31, pp. 1025-1037, 2016.

[23] Z. Zhao and $\mathrm{L}$. Wu, "Impacts of high penetration wind generation and demand response on LMPs in day-ahead market," IEEE Transactions on Smart Grid, vol. 5, pp. 220-229, 2014.

[24] J. Mohammadi, A. Rahimi-Kian, and M.-S. Ghazizadeh, "Aggregated wind power and flexible load offering strategy," IET renewable power generation, vol. 5, pp. 439-447, 2011.

[25] X. Zhang, "Optimal scheduling of critical peak pricing considering wind commitment," IEEE Transactions on Sustainable Energy, vol. 5, pp. 637-645, 2014.

[26] E. Heydarian-Forushani, M. P. Moghaddam, M. K. Sheikh-El-Eslami, M. Shafie-khah, and J. P. Catalão, "Risk-constrained offering strategy of wind power producers considering intraday demand response exchange," IEEE Transactions on sustainable energy, vol. 5, pp. 1036-1047, 2014.

[27] A. Rabiee, A. Soroudi, B. Mohammadi-Ivatloo, and M. Parniani, "Corrective voltage control scheme considering demand response and stochastic wind power," IEEE Transactions on Power Systems, vol. 29, pp. 2965-2973, 2014.

[28] "Sotavento Wind Farm [Online]. Available: http://www.sotaventogalicia.com/."

[29] N. Amjady, J. Aghaei, and H. A. Shayanfar, "Stochastic multiobjective market clearing of joint energy and reserves auctions ensuring power system security," IEEE Transactions on Power Systems, vol. 24, pp. 18411854, 2009.

[30] "Red Eléctrica de España, e-sios [Online]. Available: http://www.esios.ree.es/web-publica." 